\title{
DISTRIBUCIÓN ALTITUDINAL Y ESTACIONAL DE Dendroctonus adjunctus Blandford y Dendroctonus brevicomis Leconte EN COAHUILA, MEXICO
}

\section{ALTITUDINAL AND SEASONAL DISTRIBUTION OF Dendroctonus adjunctus Blandford AND Dendroctonus brevicomis Leconte IN COAHUILA, MÉXICO}

\author{
Cecilia G. Ruiz-González ${ }^{1}$, Jorge Méndez-González ${ }^{1}{ }^{*}$, Víctor H. Cambrón-Sandoval' \\ Mario A. García-Aranda', Juan C. Montoya-Jiménez ${ }^{1}$ y Librado Sosa-Díaz ${ }^{1}$
}

\author{
'Universidad Autónoma Agraria Antonio Narro. Buenavista Saltillo, Coahuila, México. ${ }^{2}$ Universidad Autónoma de Querétaro. Juriquilla, Querétaro, \\ México. \\ *Autor para correspondencia (jmendezg@hotmail.com)
}

\section{RESUMEN}

Los bosques de pino (Pinus spp.) de todo el mundo están declinando debido al mal manejo, al cambio climático, incendios y ataques de insectos descortezadores (Dendroctonus spp.). El objetivo de este estudio fue evaluar la abundancia altitudinal y temporal de $D$. adjunctus y $D$. brevicomis y su relación con la temperatura y características del rodal. Se establecieron dos transectos altitudinales a partir de 2600 hasta 3300 metros sobre el nivel del mar (msnm), usando trampas para insectos, cada trampa compuesta de ocho embudos, localizadas a intervalos de $100 \mathrm{~m}$ de altitud. Los insectos fueron recolectados cada dos semanas desde febrero 2015 a febrero de 2017. Los datos de temperatura y del rodal fueron obtenidos en cada sitio de muestreo. Los resultados indican que $D$. adjunctus y $D$. brevicomis fueron más abundantes por arriba de los $3000 \mathrm{msnm}$ durante primavera y verano. La abundancia de descortezadores se correlacionó significativamente con la temperatura, área basal y densidad de los árboles. En conclusión, las características del bosque y las especies de pino presentes en el rodal determinan la abundancia de $D$. adjunctus y de $D$. brevicomis en la región.

Palabras clave: Dendroctonus adjunctus, Dendroctonus brevicomis, altitud, México, temperatura.

\section{SUMMARY}

Pine forests (Pinus spp.) across the world are declining due to inappropriate management, climate change, fires, and pine beetle (Dendroctonus spp.) attacks. The objective of this study was to evaluate altitudinal and temporal abundance of $D$. adjunctus and $D$. brevicomis and its relationship with temperature and stand characteristics. Two altitudinal transects were established from 2600 to 3300 meters above sea level (masl), using insect traps, each trap made up of eight funnels, located at $100 \mathrm{~m}$ intervals of altitude. Insects were collected every two weeks from February 2015 to February 2017. Temperature and stand data were obtained at each sampling site. Results indicated that $D$. adjunctus and $D$. brevicomis were more abundant above 3000 masl during spring and summer. Bark beetle abundance was significantly correlated with temperature, basal area and tree density. In conclusion, stand characteristics and pine species present in the stand determine abundance of $D$. adjunctus and $D$. brevicomis in the region.

Key words: Dendroctonus adjunctus, Dendroctonus brevicomis, altitude, Mexico, temperature.

\section{INTRODUCCIÓN}

Los bosques albergan gran diversidad de especies, protegen el suelo, contribuyen a la estabilidad del clima, proveen de bienes y servicios y proporcionan una amplia gama de productos maderables y no maderables (Bergh y Promis, 2011). Sin embargo, el cambio climático podría favorecer el desarrollo de plagas forestales o de especies invasoras en los bosques (Pérez et al., 2007) y en este sentido se ha demostrado que la temperatura es el factor más importante debido a que influye en la supervivencia, mortalidad y en el número de generaciones de insectos, modificando la distribución geográfica de las plagas (Kocmánková et al., 2010). Así mismo, cuando los bosques se ven afectados por factores de estrés, naturales o antropogénicos, se favorece el brote de plagas (Salinas et al., 2010).

En México se cuenta con aproximadamente $42 \%$ de las especies conocidas de Pinus, su distribución altitudinal varía entre 1500 y 4000 msnm (Perry, 1991; Sánchez, 2008). Los insectos descortezadores del género Dendroctonus son huéspedes de varias de estas especies de pinos; por los daños causados se les conoce como las plagas forestales más dañinas de los bosques de pino (Rodríguez et al., 2013). Los descortezadores han causado la muerte de millones de árboles; en Canadá las pérdidas maderables han sido de hasta 2.5 millones de $\mathrm{m}^{3}$ en los últimos 20 años (Miller y Borden, 2010). En México, los daños más notables se observaron recientemente. CONAFOR (2015) registró que entre 2004 y 2014 la superficie afectada fue de 30,365 ha, atribuidas a 12 especies de insectos descortezadores. Sánchez et al. (2003) encontraron en el estado de Coahuila brotes activos de Dendroctonus spp. afectando un volumen total de $36,749 \mathrm{~m}^{3}$ de madera en rollo. Las especies de descortezadores registradas fueron: D. adjunctus Blandford, D. brevicomis LeConte, D. pseudotsugae Hopkins, D. mexicanus Hopkins, D. parallelocollis 
Chapuis y D. valens LeConte.

Dendroctonus adjunctus es considerada como una plaga primaria y $D$. brevicomis como secundaria. La primera se distribuye ampliamente en la mayoría de los sistemas montañosos y la segunda presenta distribución restringida (Salinas-Moreno et al., 2010). En México existe poca información sobre la abundancia a diferentes altitudes de estas especies. Algunos estudios relacionados con el tema fueron descritos por Salinas-Moreno et al. (2010), donde mencionan el intervalo de distribución altitudinal de 2500 a 3000 msnm y de 1680 a 3300 msnm, respectivamente. Rodríguez et al. (2010) mencionaron una distribución altitudinal de D. adjunctus de 1600 hasta 3929 msnm, con mayor incidencia de insectos en primavera y otoño. El presente estudio tuvo como objetivo evaluar la fluctuación poblacional, altitudinal y temporal de D. adjunctus y D. brevicomis y su relación con temperatura y variables dasométricas del sitio en el ejido Santa Rita, en Arteaga Coahuila.

\section{MATERIALES Y MÉTODOS}

\section{Descripción física y biológica del área de estudio}

La localidad Santa Rita, en Arteaga Coahuila, se ubica entre $25^{\circ} 17^{\prime} 36^{\prime \prime}$ y $25^{\circ} 16^{\prime} 15^{\prime \prime}$ de latitud norte y entre $100^{\circ} 30^{\prime} 52^{\prime \prime}$ y $100^{\circ} 27^{\prime} 52^{\prime \prime}$ de longitud oeste. El clima es templado subhúmedo con lluvias escasas todo el año, variando de 300 a $700 \mathrm{~mm}$ anuales; su fórmula climática es $\operatorname{BS} 1 \mathrm{~K}\left(\mathrm{x}^{\prime}\right)$, el intervalo de temperatura es de 8 a $20^{\circ} \mathrm{C}$, con rango altitudinal del área entre 1300 y 3700 msnm. El tipo de vegetación es bosque de coníferas (INEGI, 2009), cuyas principales especies son: Pinus rudis Endl, P. ayacahuite Ehren, Pseudotsuga menziesii Mirb., Abies vejarii Martínez, Ceanothus coeruleus Lag, C. buxifolius Willd y Garrya ovata Benth (Cano et al., 2007).

\section{Muestreo de insectos descortezadores}

En el sitio de estudio se establecieron dos transectos ( $A$ y $B$ ) los cuales presentaban exposición sur. Cada transecto se conformó por ocho elevaciones, una cada 100 m, iniciando desde 2600 hasta $3300 \mathrm{msnm}$. A una altura de 1.5 $m$ de un árbol no hospedero de cada elevación se colocó una trampa Lindgren ${ }^{\circledR}$ de ocho embudos con atrayente (frontalina, endo-brevicomina y alfa-pineno) y a $50 \mathrm{~m}$ en distancia horizontal se colocó una trampa sin atrayentes. El vaso colector de insectos se colgó sobre la trampa. Para conservar los insectos colectados se utilizó anticongelante marca PRESTONE AF EX al $33 \%$.

\section{Recolección de insectos, variables climáticas y datos dasométricos del sitio}

El periodo de estudio fue de dos años (febrero 2015 a febrero 2017). Las trampas se revisaron cada 15 días, resultando en un total de 52 colectas. Los insectos capturados se llevaron al laboratorio para su identificación con apoyo de un estereoscopio LEICA modelo EZA y claves taxonómicas de Cibrián et al. (1995). En un árbol cercano a la trampa con feromona se instaló un data logger modelo EL-USB-2, a una altura aproximada de $1.5 \mathrm{~m}$, el cual registró temperatura $\left({ }^{\circ} \mathrm{C}\right)$, humedad relativa $(\%)$ y punto de rocío $\left({ }^{\circ} \mathrm{C}\right)$ cada 30 minutos. La información almacenada se descargó cada seis meses usando el software EasyLogUSB. Para obtener las características del sitio se delimitó una parcela de forma cuadrada (50 × 50 m) tomando como punto central la trampa. De cada árbol se tomó la información: especie, diámetro normal $(\mathrm{cm})$, altura total $(\mathrm{m})$, diámetro de copa $(\mathrm{m})$, densidad de copa (\%), estado fitosanitario (sano e infestado), pendiente (\%) y exposición (N, S, E y O). El equipo utilizado para medir estas variables fue: distanciómetro Leica DISTO ${ }^{\mathrm{TM}}$ D810 touch, densiómetro esférico convexo (DSM43), densiómetro esférico cóncavo (DSM43A), forcípula Haglöf Mantax, cinta diamétrica (283D/10M) y GPS Garmin (GPSMAP 64).

\section{Análisis de datos}

Para caracterizar la distribución temporal y altitudinal de cada especie de descortezador, los datos de abundancia se organizaron en los siguientes niveles: por año, estación del año, altitud y transecto. Previo a la comparación y por ser información proveniente de conteos periódicos, los datos se sometieron a una prueba de normalidad de Shapiro-Wilk $(a=0.05)$; al no cumplir con este supuesto, la comparación de abundancia de insectos en cada nivel se realizó usando la prueba no paramétrica de rangos de Kruskal-Wallis (KW) al $95 \%$ de confiabilidad (Kruskal y Wallis, 1952). Para comparar la abundancia de cada especie de descortezador por año en cada transecto se usó el total de repeticiones $\sin$ importar la altitud $(n=208$ por especie por año). Entre transectos el análisis se realizó incluyendo el muestreo de ambos años sin importar la altitud ( $n=416$ repeticiones por especie). En la comparación de abundancia de descortezadores entre estaciones, las repeticiones variaron desde $n=48$ hasta 52 (por el número de semanas, pero separadas por año de evaluación y por transecto). La comparación de abundancia entre altitudes incluyó el total de muestras por sitio sin importar el año $(n=52)$. 
Para comprobar si existe relación de la abundancia (promedio de 52 fechas de colecta, ambos años) de $D$. adjunctus y $\mathrm{D}$. brevicomis en cada sitio de muestreo (altitud) con la temperatura (promedio de todos los registros de temperatura de cada 30 minutos durante dos años) y con las características del sitio (área basal por hectárea, diámetro normal promedio por sitio, altura promedio por sitio y número de árboles por hectárea) se realizó un análisis de correlación de Spearman ( $\mathrm{n}=8$ pares de datos, cada uno derivado del promedio de 52 muestreos). Los análisis estadísticos fueron realizados con el Software InfoStat 2016.

\section{RESULTADOS Y DISCUSIÓN}

\section{Fluctuación de $D$. adjunctus y $D$. brevicomis entre años y transectos}

La abundancia de $D$. adjunctus y $D$. brevicomis en el transecto A durante el primer año de evaluación (28/02/15 - 20/02/16) fue más alta ( $p=0.0178)$ que en el segundo año, con medias de 8.36 y 8.50 individuos por trampa para cada especie, mientras que en el segundo año la población fue de aproximadamente 4.5 individuos por trampa para ambas especies. En el transecto B la abundancia en ambas especies fue igual en los dos años ( $p=0.9515$ y 0.2033) (Cuadro 1). La abundancia de D. adjunctus y D. brevicomis entre transectos fue diferente $(p=0.0001)$; en el transecto
A, con 6.33 y 6.36 individuos en promedio, hubo 2 a 4 veces más individuos que en el transecto $B$, con 2.63 y 1.64 insectos, respectivamente (Cuadro 1). Esto se debe a que en el transecto A el $33.3 \%$ de las especies arbóreas son hospederas de descortezadores, y en B solo el $20.0 \%$. Tanto en A como en B, P. rudis es la especie hospedera dominante, incluso el $70 \%$ de los árboles en las altitudes de 3100 a 3200 msnm en A son de P. rudis. La temperatura promedio en los transectos A y B (9.45 y $9.32{ }^{\circ} \mathrm{C}$, respectivamente) es muy similar, por lo que la presencia de especies hospederas parece ser más importante (Cuadro 2).

Sánchez et al. (2003) reportaron brotes de D. adjunctus y $D$. brevicomis en Pseudotsuga menziesii, Abies vejarii, Pinus rudis, P. teocote Schiede ex Schltdl, P. pseudostrobus Lindl y P. arizonica Engelm en la Sierra de Arteaga Coahuila, lo cual concuerda con las especies hospederas de este estudio (P. rudis y Abies vejarii). Torres y Sánchez (2006) mencionaron que bosques densos de Pinus rudis (466 árboles ha-1), son más susceptibles a brotes de $D$. adjunctus y $D$. brevicomis debido a la competencia entre árboles y a la susceptibilidad de estrés. Los resultados obtenidos en el presente estudio comprueban que en el transecto A, con 378 árboles ha-1, hubo mayor densidad de arbolado, causando una mayor presencia de descortezadores. En Estados Unidos y Canadá las especies hospederas para D. adjunctus son P. arizonica, Pinus engelmannii Carr., P. flexilis, Pinus leiophylla Schiede ex Schltdl. \& Cham.

Cuadro 1. Prueba de Kruskal-Wallis de la abundancia de Dendroctonus adjunctus y $D$. brevicomis entre años y transectos altitudinales en Santa Rita, Arteaga Coahuila, México.

\begin{tabular}{|c|c|c|c|c|c|c|c|c|c|}
\hline Especie & & Transecto & Año & Rango & Medias & $\mathrm{Gl}$ & C & $\mathrm{H}$ & $\mathrm{p}$ \\
\hline \multicolumn{10}{|c|}{ Comparación entre años ( $n=208$ por especie) } \\
\hline \multirow{4}{*}{ D. adjunctus } & \multirow{2}{*}{\multicolumn{2}{|c|}{ A }} & $21 / 02 / 16-18 / 02 / 17$ & $196.68 b$ & 4.31 & 1 & 0.72 & 4.02 & 0.0178 \\
\hline & & & 28/02/15 - 20/02/16 & $220.32 a$ & 8.36 & & & & \\
\hline & \multirow{2}{*}{\multicolumn{2}{|c|}{ B }} & 28/02/15 - 20/02/16 & 208.79 & 3.31 & 1 & 0.64 & 0.0024 & 0.9515 \\
\hline & & & $21 / 02 / 16-18 / 02 / 17$ & 208.21 & 1.93 & & & & \\
\hline \multirow{4}{*}{ D. brevicomis } & \multirow{2}{*}{\multicolumn{2}{|c|}{$\bar{A}$}} & $21 \overline{/ 02} / 1 \overline{6-18 / 02 / 17}$ & $198.43 b$ & $\overline{4.23}$ & $\overline{1}$ & $\overline{0.74}$ & 2.92 & $0 . \overline{0477}$ \\
\hline & & & $28 / 02 / 15-20 / 02 / 16$ & $218.57 \mathrm{a}$ & 8.50 & & & & \\
\hline & \multirow{2}{*}{\multicolumn{2}{|c|}{ B }} & 28/02/15 - 20/02/16 & 213.90 & 2.28 & 1 & 0.52 & 0.84 & 0.2033 \\
\hline & & & $21 / 02 / 16-18 / 02 / 17$ & 203.10 & 1.00 & & & & \\
\hline \multicolumn{10}{|c|}{ Comparación entre transectos ( $n=416$ por especie) } \\
\hline \multirow{2}{*}{ D. adjunctus } & & B & & $399.77 b$ & 2.62 & 1 & 0.68 & 4.03 & 0.0147 \\
\hline & - & A & $\bar{L}$ & 433.23 a & 6.33 & & & & \\
\hline \multirow{2}{*}{ D. brevicomis } & & $\mathrm{B}$ & & $380.79 b$ & $\overline{1.64}$ & $\overline{1}$ & $\overline{0.64}$ & $\overline{18.37}$ & $<0 . \overline{0001}$ \\
\hline & & A & & $452.21 \mathrm{a}$ & 6.36 & & & & \\
\hline
\end{tabular}

Medias con letras iguales por columna no son estadísticamente diferentes (Kruskal-Wallis, 0.05). Gl: grados de libertad, C: factor de corrección del estadístico por observaciones empatadas, H: estadístico de la prueba no corregido por empates, p: significancia estadística. Nota: la comparación por año incluyó el muestreo en todas las altitudes en ese año; en transectos se incluyeron ambos años de muestreo en todas las altitudes. 
Cuadro 2. Características dasométricas promedio en dos transectos altitudinales (A y B) en Santa Rita, Arteaga Coahuila, México.

\begin{tabular}{|c|c|c|c|c|c|c|c|c|c|c|c|c|}
\hline \multirow{2}{*}{ Altitud } & \multirow{2}{*}{$A B\left(m^{2} h a^{-1}\right)$} & \multirow{2}{*}{$\mathrm{Dn}(\mathrm{cm})$} & \multirow{2}{*}{$H(m)$} & \multirow{2}{*}{$N\left(h a^{-1}\right)$} & \multirow{2}{*}{$\mathrm{T}\left({ }^{\circ} \mathrm{C}\right)$} & \multirow{2}{*}{ M (\%) } & \multicolumn{6}{|c|}{ Especies de coníferas (\%) } \\
\hline & & & & & & & $\mathrm{Pm}$ & $A v$ & $\mathrm{Pr}$ & $P f^{\dagger}$ & $P S^{\dagger}$ & $P C$ \\
\hline \multicolumn{13}{|c|}{ Transecto A } \\
\hline 2600 & 4.89 & 27.33 & 16.56 & 72.00 & 9.66 & 16.67 & 50.00 & 27.78 & 11.11 & 11.11 & & \\
\hline 2700 & 10.88 & 23.85 & 13.77 & 176.00 & 9.80 & 22.73 & 36.36 & 61.36 & 2.28 & & & \\
\hline 2800 & 15.97 & 28.21 & 16.36 & 216.00 & 9.78 & 48.15 & 85.19 & 5.56 & 7.40 & & 1.85 & \\
\hline 2900 & 11.52 & 17.71 & 14.49 & 384.00 & 9.28 & 41.67 & 8.33 & 56.25 & 35.42 & & & \\
\hline 3000 & 25.58 & 25.62 & 13.93 & 420.00 & 9.14 & 54.29 & 0.95 & & 23.81 & & 71.43 & 3.81 \\
\hline 3100 & 22.74 & 26.26 & 13.50 & 352.00 & 9.38 & 69.32 & & & 30.68 & & 69.32 & \\
\hline 3200 & 26.01 & 17.90 & 9.11 & 816.00 & 9.39 & 69.61 & & & 29.90 & & 70.10 & \\
\hline 3300 & 20.91 & 19.12 & 9.21 & 584.00 & 9.20 & 68.49 & 2.74 & & 47.26 & & 50.00 & \\
\hline \multicolumn{13}{|c|}{ Transecto B } \\
\hline 2600 & 1.93 & 23.39 & 11.76 & 36.00 & 10.34 & 33.33 & 33.33 & & & & 11.11 & 55.56 \\
\hline 2700 & 14.43 & 35.52 & 17.25 & 132.00 & 10.22 & 45.45 & 81.82 & 3.03 & 15.15 & & & \\
\hline 2800 & 9.19 & 26.58 & 12.50 & 124.00 & 10.15 & 25.81 & 48.39 & 25.81 & 12.90 & & 12.90 & \\
\hline 2900 & 19.58 & 20.53 & 10.02 & 508.00 & 9.52 & 41.73 & & 28.35 & 70.08 & & 1.57 & \\
\hline 3000 & 12.31 & 25.17 & 11.47 & 180.00 & 9.04 & 31.11 & 2.22 & 20.00 & 75.56 & & 2.22 & \\
\hline 3100 & 21.21 & 18.77 & 12.40 & 616.00 & 8.69 & 35.06 & 11.04 & 59.09 & 27.92 & & 1.95 & \\
\hline 3200 & 19.18 & 22.77 & 12.13 & 344.00 & 8.81 & 40.70 & 53.49 & & 40.70 & & 5.81 & \\
\hline 3300 & 48.29 & 27.03 & 17.04 & 744.00 & 7.78 & 70.97 & 29.57 & & 16.13 & & 54.30 & \\
\hline
\end{tabular}

AB: área basal, Dn: diámetro normal, H: Altura total, N: número de árboles, T: temperatura promedio, M: mortalidad, Pm: Pseudotsuga menziesii, Av: Abies vejarii, Pr: Pinus reflexa, Pf: Pinus flexilis, Ps: Pinus rudis, Pc: Pinus cembroides, $\left({ }^{+}\right)$: especies hospederas.

Pinus ponderosa Douglass ex Lawson, Pinus strobiformis Engelm, mientras que D. brevicomis se presenta en Pinus coulteri D. Don y P. ponderosa (Bentz et al., 2010). En Coahuila las especies afectadas son $P$. rudis, $P$. flexilis y Abies vejarii; aunado a esto, se ha pronosticado que el aumento de temperatura (de 3 a $5^{\circ} \mathrm{C}$ ) para Estados Unidos y Canadá propiciará aumento en las poblaciones de plagas (Dukes et al., 2009).

\section{Fluctuación estacional de $D$. adjunctus y $D$. brevicomis}

La abundancia de $D$. adjunctus fue estadísticamente diferente entre estaciones del año ( $p<0.0001)$, siendo mayor en primavera del 2015 en ambos transectos con 19.46 (transecto A) y 8.35 (transecto B) insectos en promedio y casi nula en otoño del 2015 ( $n=0.21$ en transecto $A$ y $n=0$ en B) (Figura 1 A y 1 C). D. brevicomis presentó un patrón similar; las estaciones de mayor vuelo de descortezadores fueron verano del 2015 (transecto A) y primavera del 2015 (transecto B) promediando 15.64 y 6.13 insectos, respectivamente en cada estación. Otoño del 2015 y 2016 fueron las estaciones donde hubo menor presencia de $D$. brevicomis ( $\mathrm{n}=0.94$ transecto $A$ y $n=0.33$ transecto $B$ ) (Figura 1 $B$ y 1 D). En el transecto $A$ las temperaturas más altas se registraron en primavera y verano del 2015, siendo de 10 y $13^{\circ} \mathrm{C}$, respectivamente, ocurriendo aquí la mayor abundancia de insectos. En las estaciones con menor presencia de descortezadores (otoño 2015 y 2016) las temperaturas fueron de 9.10 y $8.83^{\circ} \mathrm{C}$ (Figura 2 A); para el año 2016 se presentó un patrón similar en la abundancia de descortezadores (Figura 1 A y 1 B).

Primavera del 2015 se caracterizó por presentar temperaturas de hasta $13^{\circ} \mathrm{C}$ y alta abundancia de descortezadores de hasta 400 en total (Figura 2 B), la mayor abundancia registrada en esta estación se debe a las temperaturas altas, ya que en las estaciones con menor presencia de descortezadores la temperatura fue menor a $10{ }^{\circ} \mathrm{C}$. Los resultados obtenidos concuerdan con Sánchez-Martínez et al. (2008) y Rodríguez et al. (2010), al reportar primavera como la estación de mayor incidencia de descortezadores ( $n=20$ y 6.53 en promedio, respectivamente) en $P$. arizonica y $P$. hartewgii Lindl. Estos autores mencionan a otoño con alta incidencia de descortezadores, lo cual no concuerda con este estudio, ya que aquí otoño fue la estación con menor número de individuos. En el estado de Veracruz, en cambio, se observaron hasta 170 individuos de D. adjunctus en $P$. rudis durante la primavera (Rodríguez et al., 

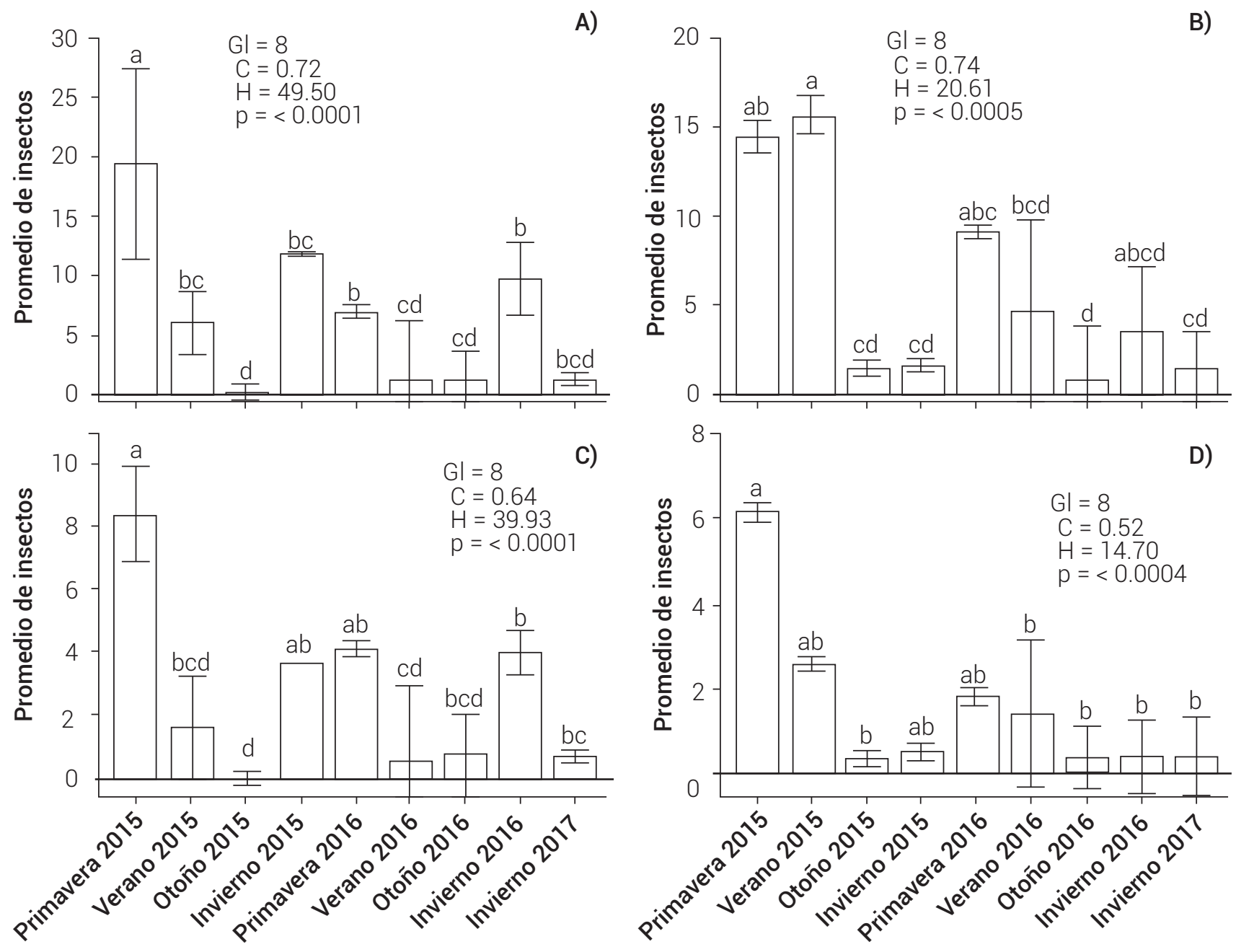

Figura 1. Prueba de rangos de Kruskal-Wallis de la fluctuación temporal en el tamaño de población de Dendroctonus adjuntus (A y C) y D. brevicormis (B y D) en los transectos A (A y B) y B (C y D) en Santa Rita, Arteaga Coahuila, México. Las barras indican los valores medios ( \pm error estándar). Medias con letras iguales (minúsculas) no son estadísticamente diferentes (Kruskal-Wallis, 0.05). Gl: grados de libertad, C: factor de corrección del estadístico por observaciones empatadas, $\mathrm{H}$ : estadístico de la prueba no corregida por empates, $\mathrm{p}$ : significancia estadística.

2013). Por su parte, Vázquez et al. (2007) mencionaron que la mayor fluctuación de D. mexicanus en Michoacán (ambos estudios en México) se presentó de marzo a junio, durante actividades de aprovechamiento, cuando los árboles fueron más susceptibles a ataques de insectos.

\section{Fluctuación altitudinal de $D$. adjunctus y $D$. brevicomis}

La prueba de Kruskal-Wallis demostró que la mayor abundancia de $D$. adjunctus en ambos transectos (A y B) ocurre a los 3300 msnm ( $n=12.62$ transecto $A$ y $n=9.75$ transecto B) (Figura 3 A y 3 C). D. brevicomis mostró un patrón similar, mayor en el transecto A, a los 3000 msnm y en el transecto $\mathrm{B}$ a los 3300 msnm con medias de 18.92 y 4.62 insectos, respectivamente (Figura 3 B y 3 D). La menor cantidad de insectos de ambas especies ocurrió de los 2600 a 2800 msnm. El intervalo altitudinal de 3000 a 3300 m se caracterizó por presentar alto porcentaje de la especie hospedera (P. rudis), mayor densidad del arbolado (400 a 800 árboles $\mathrm{ha}^{-1}$ ) en ambos transectos y alto porcentaje de mortalidad de árboles (hasta $70 \%$ en ambos transectos) (Cuadro 2).

Los resultados obtenidos concuerdan con los intervalos de distribución altitudinal reportados por Salinas-Moreno et al. $(2004 ; 2010)$ en México (D. adjunctus en intervalo de 3100 a 3500 msnm y D. brevicomis de 1680 a 3300 msnm). Estos autores también reportaron el mayor porcentaje de incidencias de D. adjunctus en P. hartwegii (58 a $83.3 \%$ ) y D. brevicomis en $P$. durangensis Martínez y $P$. engelmannii (31 a $40 \%$ ), lo cual no coindice con las especies hospederas 


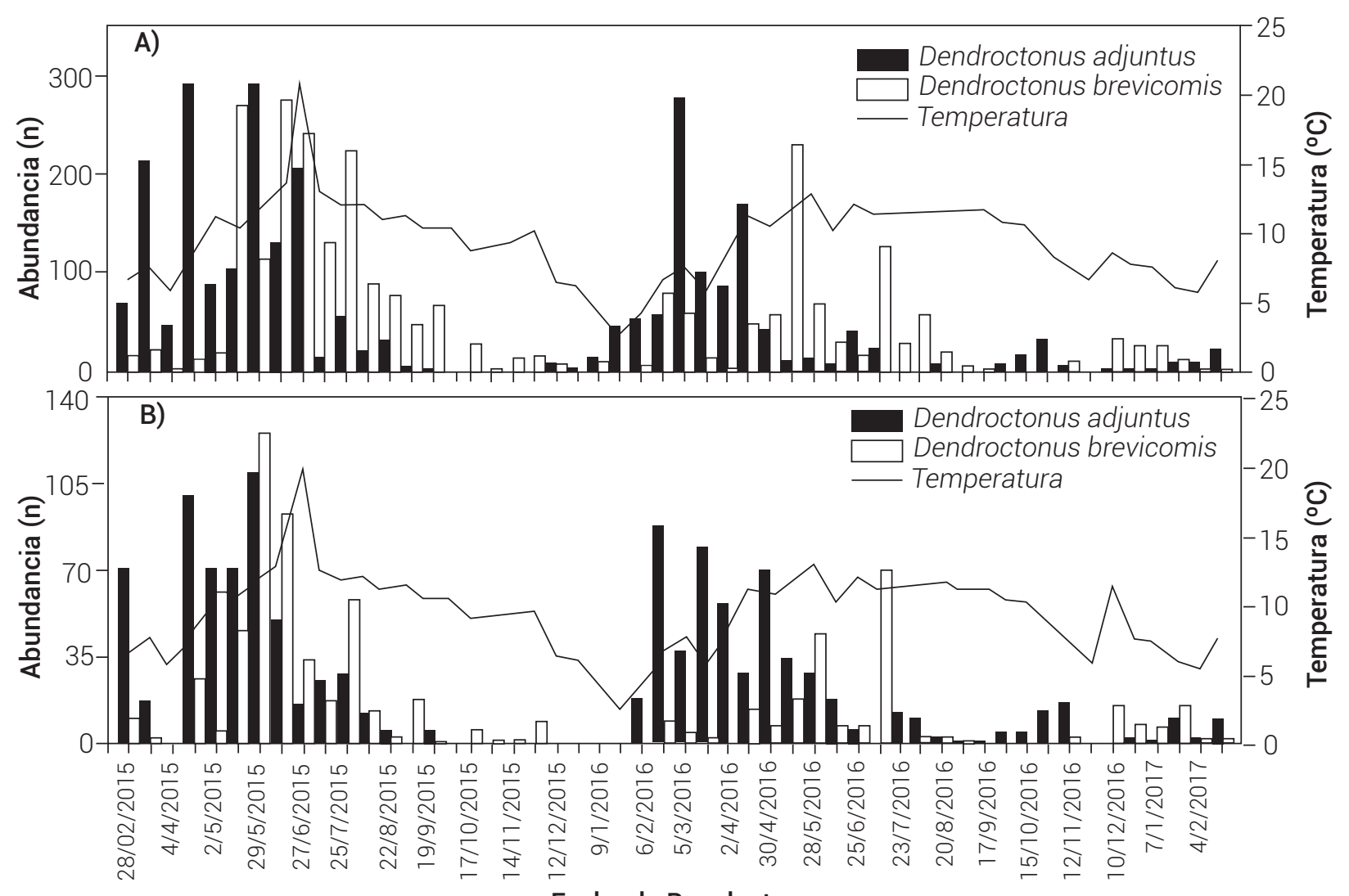

Fecha de Recolecta

Figura 2. Fluctuación poblacional total de Dendroctonus adjunctus y $D$. brevicomis por fecha de colecta y su relación con temperatura promedio en los transectos A (A) y B (B) en Santa Rita, Arteaga Coahuila, México. Nota: en el eje "Y" se representa la abundancia total registrada en todas las altitudes en cada fecha de colecta. La temperatura es el promedio aritmético de todos los datos registrados por los sensores en todas las altitudes durante el periodo de una fecha de colecta a la siguiente, en cada transecto.

de este estudio. Los brotes de D. adjunctus en la Sierra Raspadura, Chihuahua se presentaron entre 2560 y 2860 msnm, debido a la mayor distribución de especies hospederas (P. arizonica) y altas densidades de Pinus (109 árboles ha-1) (Sánchez y Silva, 2008). En Arizona, U.S.A. el rango de distribución para $D$. adjunctus se encuentra aproximadamente entre 2500 y 2700 msnm y $D$. brevicomis entre 2050 y 2250, lo cual varía con el presente estudio, debido a la ubicación en la que se hizo el muestreo en este trabajo; de la misma forma, la mayor abundancia de insectos se atribuye a mayor densidad de especies hospederas (Pinus ponderosa) y temperaturas máximas de más de $14.5^{\circ} \mathrm{C}$ (Hayes et al., 2008; Williams et al., 2008).

\section{Relación de $D$. adjunctus y $D$. brevicomis con temperatura y variables del sitio}

Las correlaciones entre la abundancia de $D$. adjunctus y D. brevicomis en los sitios a lo largo de los transectos fueron significativas $(r>0.70$ y $p<0.03)$ en ambos transectos.
La abundancia de ambas especies de descortezadores se correlacionó positiva y significativamente con el área basal $(r>0.70$ y $p<0.05)$, con el número de árboles por hectárea $(r>0.76$ y $p<0.04)$, pero negativamente con la temperatura promedio en los sitios $(r>0.60$ y $p<0.08)$ (Cuadro 3). Los resultados obtenidos en el presente estudio son similares a los de Sánchez y Silva (2008), donde mencionan correlaciones significativas entre la abundancia de insectos con el área basal ( $r=0.806 ; p>0.0001)$. Hayes et al. (2008) demostraron que entre más área basal exista mayor será la abundancia y mayor el ataque de Dendroctonus. Los resultados obtenidos y los reportados por otros autores han demostrado que las características de los sitios de estudio (altitud, área basal, diámetro, especies hospederas y la densidad del arbolado) influyen en la fluctuación de los insectos descortezadores.

\section{CONCLUSIONES}

La mayor abundancia de $D$. adjunctus y $D$. brevicomis 

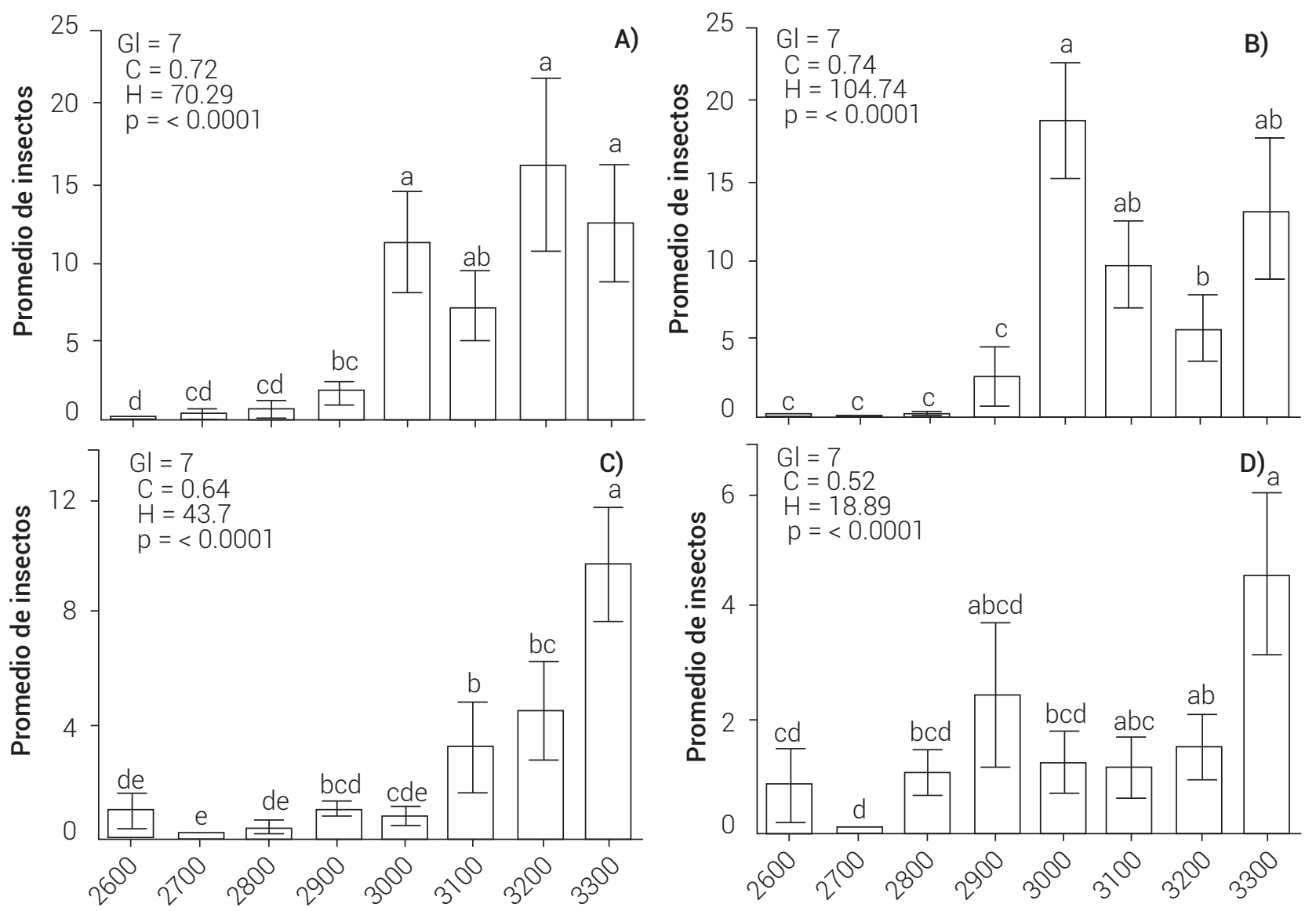

Altitud (msnm)

Altitud (msnm)

Figura 3. Prueba de Kruskal-Wallis de los valores promedio del número de insectos de Dendroctonus adjunctus (A y $C)$ y $D$. brevicomis (B y D) a lo largo del gradiente altitudinal de los transectos A (A y B) y B (C y D) en Santa Rita, Arteaga Coahuila, México. Las barras indican medias ( \pm error estándar). Medias con letras iguales (minúsculas) no son estadísticamente diferentes (Kruskal-Wallis, 0.05). Gl: grados de libertad, C: factor de corrección del estadístico por observaciones empatadas, $\mathrm{H}$ : estadístico de la prueba no corregido por empates; $\mathrm{p}$ : significancia estadística.

Cuadro 3. Correlación entre variables del sitio y temperatura con Dendroctonus adjunctus y $D$. brevicomis $(n=8$, promedio de 52 colectas) en dos transectos altitudinales en Santa Rita, Arteaga Coahuila, México.

\begin{tabular}{|c|c|c|c|c|c|c|c|}
\hline & & $A B\left(m^{2} h a^{-1}\right)$ & $\mathrm{Dn}(\mathrm{cm})$ & $\mathrm{H}(\mathrm{m})$ & $\mathrm{Nha}^{-1}$ & $\mathrm{~T}\left({ }^{\circ} \mathrm{C}\right)$ & D. adjunctus \\
\hline \multicolumn{8}{|l|}{ Transecto A } \\
\hline \multirow{2}{*}{ D. adjunctus } & r & 0.9048 & -0.5238 & -0.8095 & 0.9762 & -0.6429 & \\
\hline & $p$ & 0.0167 & 0.1658 & 0.0322 & 0.0098 & 0.0890 & \\
\hline \multirow{2}{*}{ D. brevicomis } & r & 0.8024 & -0.2515 & -0.5270 & 0.7785 & -0.8862 & 0.8264 \\
\hline & $p$ & 0.0165 & 0.5479 & 0.1796 & 0.0229 & 0.0034 & 0.0114 \\
\hline \multicolumn{8}{|l|}{ Transecto B } \\
\hline \multirow{2}{*}{ D. adjunctus } & r & 0.7143 & -0.4286 & -0.1190 & 0.7619 & -0.7857 & \\
\hline & $\mathrm{p}$ & 0.0588 & 0.2568 & 0.7528 & 0.0438 & 0.0376 & \\
\hline \multirow{2}{*}{ D. brevicomis } & r & 0.6905 & -0.2619 & -0.3095 & 0.7857 & -0.7619 & 0.7857 \\
\hline & $\mathrm{p}$ & 0.0677 & 0.4884 & 0.4128 & 0.0376 & 0.0438 & 0.0376 \\
\hline
\end{tabular}

R: coeficiente de correlación de Spearman, p: significancia estadística, AB: área basal, Dn: diámetro normal promedio del sitio, H: altura total promedio del sitio, N: número de árboles, T: temperatura promedio. Nota. La abundancia usada en el análisis representa el promedio de 52 muestras comparado con el valor de las variables dasométricas y ambientales en cada sitio. 
ocurre en primavera y verano debido a las altas temperaturas que se presentan en estas estaciones del año. La menor presencia ocurre en otoño e invierno. D. adjunctus se registró principalmente en la altitud de 3300 msnm, mientras que D. brevicomis entre 3000 y 3300 msnm, debido a las condiciones ambientales (microclima) y al número de especies hospederas que se presenta en cada altitud. La abundancia de insectos de D. adjunctus y D. brevicomis se correlacionó significativamente con el área basal, el número de árboles y la temperatura promedio del sitio. Queda de manifiesto que los brotes de descortezadores se pueden prevenir mediante tratamientos silvícolas, disminuyendo la densidad del arbolado y manejando el área basal para reducir la competencia entre las especies arbóreas existentes en el rodal.

\section{AGRADECIMIENTOS}

Proyecto de investigación financiado por el Fondo Sectorial CONAFOR-CONACYT-2014, C01-234547.

\section{BIBLIOGRAFÍA}

Bentz B. J., J. Régniére, C. J. Fettig, E. M. Hansen, J. L. Hayes, J. A. Hicke, ... and S. J. Seybold (2010) Climate change and bark beetles of the Western United States and Canada: Direct and indirect effects. Bioscience 60: 602-613, https://doi.org/10.1525/ bio.2010.60.8.6

Bergh G. y A. Promis (2011) Conservación de los bosques nativos de Chile un análisis al Informe FAO sobre la Evaluación de los Recursos Forestales Nacionales. Revista Bosque Nativo 48: 9-11.

Cano P. A., O. U. Martínez B. y A. Quiñonez Ch. (2007) Caracterización y diagnóstico del medio físico de la microcuenca Santa Rita del municipio de Arteaga, Coahuila. INIFAP-CIRNE. Campo Experimental Saltillo. Publicación Especial Núm. 13. Coahuila, México. $44 \mathrm{p}$.

Cibrián T. D., J. T. Méndez M., R. Campos B., H. O. Yates III. y J. E. Flores L. (1995) Insectos Forestales de México/Forest Insects of México. Universidad Autónoma Chapingo, Estado de México, México. $453 p$.

CONAFOR, Comisión Nacional Forestal (2015) Superficie forestal afectada por plagas y enfermedades forestales. Base de Datos Estadísticos-Badesniarn. http://dgeiawf.semarnat.gob.mx:8080/ approot/dgeia_mce/html/mce_index.html?De=BADESNIARN. (Julio 2016)

Dukes J. S., J. Pontius, D. Orwig, J. R. Garnas, V. L. Rodgers, N. Brazee, ... and M. Ayres (2009) Responses of insect pests, pathogens, and invasive plant species to climate change in the forests of northeastern North America: What can we predict? Canadian Journal of Forest Research 39:231-248, http://10.1139/X08-171

Hayes Ch. J., T. E. DeGomez, K. M. Clancy, K. K. Williams, J. D. McMillin and J. A. Anhold (2008) Evaluation of funnel traps for characterizing the bark beetle (Coleoptera: Scolytidae) communities in Ponderosa Pine forests of North-Central Arizona. Journal of Economic Entomology 101:1253-1265. http://dx.doi.org/10.1603/00220493(2008)101[1253:EOFTFC]2.0.CO;2

INEGI, Instituto Nacional de Estadística, Geografía e Informática (2009) Prontuario de información geográfica municipal de los Estados Unidos Mexicanos Arteaga, Coahuila de Zaragoza. Clave geoestadística 05004. http://www3.inegi.org.mx/sistemas/mexicocifras/datos-geograficos/05/05004.pdf.

Kocmánková E., M. Trnka, J. Eitzinger, H. Formayer, M. Dubrovský, D. Semerádová, ... and M. Možný (2010) Estimating the impact of climate change on the occurrence of selected pests in the Central European region. Climate Research 44:95-105, http://10.3354/ cr00905

Kruskal W. H. and A. Wallis W. (1952) Use of ranks in one-criterion variance analysis. Journal of the American Statistical Association 47:583-621, http://10.2307/2280779

Miller D. R. and J. H. Borden (2010) Dose-dependent and species-specific responses of pine bark beetles (Coleoptera: Scolytidae) to monoterpenes in association with pheromones. The $\mathrm{Ca}$ nadian Entomologist 132:183-195, https://doi.org/10.4039/ Ent132183-2

Pérez C. J., B. Locatelli, R. Vignola y P. Imbach B. (2007) Importancia de los bosques tropicales en las políticas de adaptación al cambio climático. Revista Recursos Naturales y Ambiente 51-52:4-11.

Perry J. P. (1991) The Pines of Mexico and Central America. Timber Press. Portland, Oregon, USA. 231 p.

Rodríguez O. A., A Equihua M., J. Cibrián T., E. G. Estrada V., J. T. Méndez M., J. Villa C. y R. M. Barrón Y. (2013) Fluctuación de Dendroctonus adjunctus (Coleoptera: Curculionidae: Scolytinae) y sus depredadores atraídos por frontalina + alfa-pineno, en los Pescados, Veracruz, México. Revista Chilena de Entomología 38:41-50.

Rodríguez O. A., A. Equihua M., J. Cibrián T. y E. G. Estrada V. (2010) Fluctuación de Dendroctonus adjunctus Blandford (Curculionidae: Scolytinae) y sus depredadores atraídos por frontalina + alfapineno, en la estación experimental de Zoquiapan, Edo. de México. Boletín del Museo de Entomología de la Universidad del Valle 11:20-27.

Salinas M. Y., A. Ager, C. F. Vargas, J. L. Hayes and G. Zúñiga (2010) Determining the vulnerability of Mexican pine forests to bark beetles of the genus Dendroctonus Erichson (Coleoptera: Curculionidae: Scolytinae). Forest Ecology and Management 260:52-61. http://10.1016/j.foreco.2010.03.029

Salinas-Moreno Y., M. G. Mendoza, M. A. Barrios, R. Cisneros, J. Macías-Samano and G. Zúniniga (2004) Areography of the genus Dendroctonus (Coleoptera: Curculionidae: Scolytinae) in Mexico. Journal of Biogeography 31:1163-1177. https://doi.org/10.1111/j.13652699.2004.01110.x

Salinas-Moreno Y., C. F. Vargas M., G. Zúñiga, J. Víctor, A. Ager y J. L. Hayes (2010) Atlas de distribución geográfica de los descortezadores del género Dendroctonus (Curculionidae: Scolytinae) en México. Instituto Politécnico Nacional, Comisión Nacional Forestal. $90 \mathrm{p}$.

Sánchez G. A. (2008) Una visión actual de la diversidad y distribución de los pinos de México. Madera y Bosques 14:107-120.

Sánchez M. G. y S. Silva R. (2008) Caracterización de un brote de Dendroctonus adjunctus registrado en el estado de Chihuahua. INIFAP. Campo Experimental Pabellón. Folleto técnico Núm. 38. Aguascalientes, México. 48 p.

Sánchez S. J. A., L. M. Torres E., A. Cano P. y 0. U. Martínez B. (2003) Daños y diversidad de insectos descortezadores de coníferas del Noreste de México. Revista Mexicana de Ciencias Forestales 28:41-56

Sánchez-Martínez G., R. Narváez-Flores y J. A Olivo-Martínez (2008) Ciclo biológico y patrón estacional del descortezador de las alturas (Dendroctonus adjunctus Blanford) en la Sierra la Raspadura, Chihuahua. INIFAP. Campo Experimental Pabellón. Folleto técnico Núm. 37. Aguascalientes, México. 31 p.

Torres E. L. M. y J. A. Sánchez S. (2006) Determinación de la fluctuación estacional de Dendroctonus adjunctus Blandford en Pinus rudis mediante el uso de feromonas. INIFAP-CIRNE. Campo Experimental Saltillo. Folleto Técnico Núm. 27, Coahuila, México. $39 \mathrm{p}$.

Vázquez C. I., G. Sánchez M. y S. Madrigal H. (2007) Fluctuación poblacional de Dendroctonus mexicanus Hopk., bajo dos condiciones de manejo forestal en Michoacán, México. Revista Mexicana de Ciencias Forestales 32:57-77.

Williams K. K., J. D. McMillin, T. E. DeGomez, K. M. Clancy and A. Miller (2008) Influence of elevation on bark beetle (Coleoptera: Curculionidae, Scolytinae) community structure and flight periodicity in Ponderosa pine forests of Arizona. Environmental Entomology 37:94-109, http://dx.doi.org/10.1603/0046225X(2008)37[94:IOEOBB]2.0.C0;2 